\title{
REVISED A novel ultrasound technique to detect early chronic
}

\section{kidney disease [version 2; peer review: 2 approved]}

\author{
Dulitha K. Hewadikaram (D1), Mudhitha Bandara², Amal N. Pattivedana2, \\ Hiran H. E. Jayaweera², Kithsiri M. Jayananda², W. A. Monica Madhavi², \\ Aruna Pallewatte ${ }^{3}$, Channa Jayasumana ${ }^{4}$, Sisira Siribaddana (D)4, \\ Janaka P. Wansapura (iD)2 \\ ${ }^{1}$ General Sir John Kotelawala Defence University, Rathmalana, Sri Lanka \\ 2University of Colombo, Colombo, Sri Lanka \\ ${ }^{3}$ National Hospital of Sri Lanka, Colombo, Sri Lanka \\ ${ }^{4}$ Rajarata University of Sri Lanka, Anuradhapura, Sri Lanka
}

V2 First published: 11 Apr 2018, 7:448

https://doi.org/10.12688/f1000research.14221.1

Latest published: 24 Feb 2019, 7:448

https://doi.org/10.12688/f1000research.14221.2

\section{Abstract}

Chronic kidney disease (CKD) of unknown etiology is recognized as a major public health challenge and a leading cause of morbidity and mortality in the dry zone in Sri Lanka. CKD is asymptomatic and are diagnosed only in late stages. Evidence points to strong correlation between progression of CKD and kidney fibrosis. Several biochemical markers of renal fibrosis have been associated with progression of CKD. However, no marker is able to predict CKD consistently and accurately before being detected with traditional clinical tests (serum creatinine, and cystatin C, urine albumin or protein, and ultrasound scanning).

In this paper, we hypothesize that fibrosis in the kidney, and therefore the severity of the disease, is reflected in the frequency spectrum of the scattered ultrasound from the kidney. We present a design of a simple ultrasound system, and a set of clinical and laboratory studies to identify spectral characteristics of the scattered ultrasound wave from the kidney that correlates with CKD. We believe that spectral parameters identified in these studies can be used to detect and stratify CKD at an earlier stage than what is possible with current markers of CKD.

Keywords

Chronic Kidney Disease of unknown etiology, Ultrasound spectral characteristics, Kidney fibrosis

\section{Open Peer Review Approval Status \\ 1 \\ 2 \\ version 2 \\ (revision) \\ 24 Feb 2019 \\ version 1 \\ 11 Apr 2018

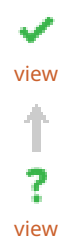 \\ 1. Gaetano Lucisano, Imperial College Renal and Transplant Centre, London, UK \\ 2. Federico Nalesso $[D$, Università di Padova, Padova, Italy \\ Any reports and responses or comments on the article can be found at the end of the article.}


Corresponding author: Janaka P. Wansapura (janaka.wansapura@phys.cmb.ac.lk)

Author roles: Hewadikaram DK: Writing - Original Draft Preparation; Bandara M: Investigation, Resources; Pattivedana AN: Methodology; Jayaweera HHE: Resources; Jayananda KM: Resources; Madhavi WAM: Resources; Pallewatte A: Methodology, Resources; Jayasumana C: Conceptualization; Siribaddana S: Resources, Writing - Review \& Editing; Wansapura JP: Conceptualization, Funding Acquisition

Competing interests: No competing interests were disclosed.

Grant information: Funding by National Research Council, Vidya Mawatha, Sri Lanka (NRC 16-044).

The funders had no role in study design, data collection and analysis, decision to publish, or preparation of the manuscript.

Copyright: $\odot 2019$ Hewadikaram DK et al. This is an open access article distributed under the terms of the Creative Commons Attribution License, which permits unrestricted use, distribution, and reproduction in any medium, provided the original work is properly cited.

How to cite this article: Hewadikaram DK, Bandara M, Pattivedana AN et al. A novel ultrasound technique to detect early chronic kidney disease [version 2; peer review: 2 approved] F1000Research 2019, 7:448 https://doi.org/10.12688/f1000research.14221.2

First published: 11 Apr 2018, 7:448 https://doi.org/10.12688/f1000research.14221.1 


\section{REVISED Amendments from Version 1}

We agree with the reviewer's concerns with regards to the sensitivity analysis and have added this information to the 'Proposed ex vivo experiment' section.

Additionally we have stated that all clinical results will be normalized to anthropometric features such as body height etc. as highlighted by Lucisano et al., 2015.

\section{See referee reports}

\section{Introduction}

Chronic kidney disease (CKD) is a major public health challenge and a leading cause of morbidity and mortality ${ }^{1}$. About $8-16 \%$ of the world population is affected by $\mathrm{CKD}^{2-4}$ with increased risk for end-stage renal disease, cardiovascular disease, and death ${ }^{5}$. To date, no specific treatment has shown to arrest the progression of CKD, except dialysis or kidney transplantation ${ }^{1}$. Considering the high cost of renal replacement therapy, the growing prevalence of CKD has implications for health and social care systems ${ }^{6}$ especially for developing nations. New variety of CKD has been identified among paddy farmers (known as $\mathrm{CKD}$ of unknown etiology $(\mathrm{CKDu})$ or Chronic Interstitial Nephropathy among Agricultural Communities (CINAC)) in the North Central Province of Sri Lanka ${ }^{7}$. One fifth of the population in Anuradhapura, Polonnaruwa and Badulla districts suffer from $\mathrm{CKDu}$ and it has already become a major public health issue in Sri Lanka ${ }^{8}$

CKD is silent killer because it starts insidiously and progresses slowly until end stage renal disease. The main challenges to improve outcomes in patients with CKD are the inability to identify patients with CKD in early subclinical stages ${ }^{9-11}$.

Evidence suggests kidney fibrosis occurs in every type of CKD and leads to progressive and irreversible loss of renal function ${ }^{12,13}$. Progressive deposition of extracellular matrix in glomeruli (glomerulosclerosis) and/or interstitial space (tubulointerstitial fibrosis) is known as kidney fibrosis. Recent studies have hypothesized several biomarkers of renal fibrosis. Among them are, transforming growth factor- $\beta 1$ (TGF- $\beta 1$ ) a pro-fibrotic cytokine measured in urine and serum, bone morphogenetic protein-7 (BMP-7) recognized as a natural antagonist to TGF- $\beta 1$ measured in serum and epidermal growth factor (EGF), a tubule-specific protein critical for cell differentiation and regeneration measured in urine. These markers of renal fibrosis have been associated with progression of CKD as measured by eGFR. However, no marker is able to predict CKD before being detected with traditional clinical tests (serum creatinine, and cystatin $\mathrm{C}$, urine albumin, and ultrasound scanning) consistently and accurately. New biomarkers point to generalized processes that cause fibrosis, but they do not directly reflect kidney pathology. Biomarkers measured in serum or urine from CKD patients may not reflect the degree of kidney fibrosis and these should be corroborated with actual pathological measures of the kidney fibrosis in order to predict a given patient's outcome.

In the context of quantification of fibrosis, several different techniques such as renal biopsy, magnetic resonance imaging
(MRI), ultrasound (US) scanning can be utilized ${ }^{14,15}$. However, there are some limitations when it comes to renal biopsy as it does not cover the entire organ. The extent of fibrosis can be quantified by renal biopsy but it is an invasive procedure with associated risks ${ }^{16}$.

MRI is ideally suited to quantify fibrosis via late Gadolinium enhancement and relaxation time measurement ${ }^{14,15,17}$. Previously, we have shown that young kidney patients on maintenance dialysis develop myocardial fibrosis quantifiable via MRI T2 relaxation time ${ }^{15}$. But MRI is an expensive modality, which is not readily accessible to the general population at risk of CKDu in Sri Lanka.

Fibrosis decreases the elasticity of tissue, hence measures of tissue elasticity is a surrogate marker of fibrosis. There are several techniques to measure elasticity in tissue using US. Among them transient elastography is the most common and widely used method. It measures tissue deformation while applying external pressure to the organ. Due to its retroperitoneal position this is not feasible in the kidney though it has been successfully used to quantitate liver fibrosis.

Acoustic radiation force impulse (ARFI) imaging and shear wave velocity (SWV) are two other US elastography techniques. However, the reliability of these techniques in measuring kidney fibrosis has not been consistent ${ }^{18-20}$. Recent feasibility studies show that both ARFI and SWV failed to correlate with kidney fibrosis ${ }^{19,20}$. Unlike liver, the kidney is not homogenous in tissue character; it is more perfused, with two distinct zones and pathologically more complex. Therefore in our opinion, and as evident by these studies, elasticity of the kidney is probably a less reliable surrogate for kidney fibrosis.

\section{Hypothesis}

Our approach to developing a non-invasive imaging method to detect early signs of CKD is based on the following hypothesis:

Disease severity in CKD is associated with changes in the Fourier transform of the scattered ultrasound waves (Radio Frequency Spectrum) from the cortex of the CKD kidney.

The rationale for our hypotheses is based on the ultrasound physics.

The speckle patterns in B-Mode ultrasound images is the result of interference of scattered ultrasound waves from scatterers whose size is much smaller than the ultrasound wave length. B-mode ultrasound images are constructed from the amplitude modulation of the time domain scattered signal, known as the Radio Frequency echo (RF echo). In B-mode ultrasound the frequency dependent information of the RF echo is not utilized. However, theoretical and phantom studies ${ }^{21}$ have shown that the frequency spectrum of the RF echo (RF spectrum), can be related to microstructural properties such as shape, size, density and acoustical properties of tissue. Thus, changes in scatterer properties in tissue may affect the RF spectrum. On the other hand, due to its size and structure, accumulation of fibrosis could change the scatterer properties of tissue. 
Thus, it is plausible that increasing fibrosis in the CKD kidney, and therefore the severity of the disease, is associated with changes in the RF spectrum. This hypothesis is reinforced by studies in the liver showing correlation between B-mode ultrasound features and the presence of fibrosis ${ }^{22-24}$.

A simple ultrasound system capable of RF echo signal acquisition and spectral analysis will be constructed to perform ex vivo and clinical studies. In the ex vivo experiment, correlations between the RF spectrum, speckle patterns and tissue characteristics will be investigated. In the clinical study, correlations between the RF spectrum, speckle pattern and the CKD stage will be investigated. In both cases speckle patterns will be used as the link between RF spectrum and fibrosis because speckle patterns are known to correlate with fibrotic stage in the liver ${ }^{22-24}$.

\section{The portable US system}

Majority of the affected population consist of rural farmers who cannot afford to undergo regular medical screenings. Here we present an affordable and portable ultrasound system with the proposed technology that can be taken to the people at risk, where they live.

The proposed portable ultrasound probe system will have five major components. They are: waveform generator, high voltage amplifiers, a controlling unit consist of a Field Programmable Gate Array (FPGA) and an ARM microcontroller, ultrasound transducer, and an analog-to-digital converter. A block diagram of the system is shown in Figure 1.

The controller will initiate a series of $1-10 \mathrm{MHz}$ high voltage pulses through the ultrasonic transducer. The echo received by the same transducer, after suitable amplification and filtering will be digitized at the rate of $125 \mathrm{MS} / \mathrm{s}$. This data will be processed by the microcontroller and then will be transmitted to a laptop computer via Wi-Fi for further analysis and display.
A single element immersion transducer (Olympus V310-SU $5 \mathrm{MHz}$ and V312-SU $10 \mathrm{MHz}$ ) will be used. Data analysis software will be developed and installed on the PC.

It is estimated that a prototype of the proposed US system can be built for approximately USD 1400.

\section{Proposed ex vivo experiment}

In the laboratory experiment, RF spectrum and speckle pattern analysis will be performed on a range of ex vivo tissue (e.g. bovine liver, kidney tissue, etc.). The tissue sample will be completely submerged in degassed water $(0.9 \%$ saline solution) and a $5 \mathrm{MHz}$ or $10 \mathrm{MHz}$ single element 0.25 in elemental diameter; immersion transducer (Olympus-V310-SU, Band width $16-24 \mathrm{MHz}$ at $-6 \mathrm{~dB}$ ) will be used for scanning. Tissue sample will be held stationary and the transducer will move 200-micrometer step using computer controlled micro position system. RF echo data will be acquired using the proposed US system. A Hamming window will be applied to RF signal followed by fast Fourier transform (FFT) to calculate the RF power spectrum ${ }^{21}$. The RF spectrum will be normalized by a spectrum obtained from a standard phantom with identical acquisition parameters. The calibrated power spectrum will be fitted to a linear model over its bandwidth. This will give the standard spectral parameters: spectral intercept $(\mathrm{dB}$; extrapolation to zero frequency) and spectral slope. In addition to the standard spectral parameters we will explore other statistical measures of the spectrum in order to identify attributes of the RF spectrum that are most sensitive to tissue characteristics.

The RF data from different ex vivo samples will be used to construct a B-Mode ultrasound image of the sample. This image will be analyzed for speckle features employing standard techniques such as statistical methods, model-based approaches, signal processing and geometrical analysis ${ }^{25}$. The speckle feature parameters will be tested on their sensitivity to differentiate

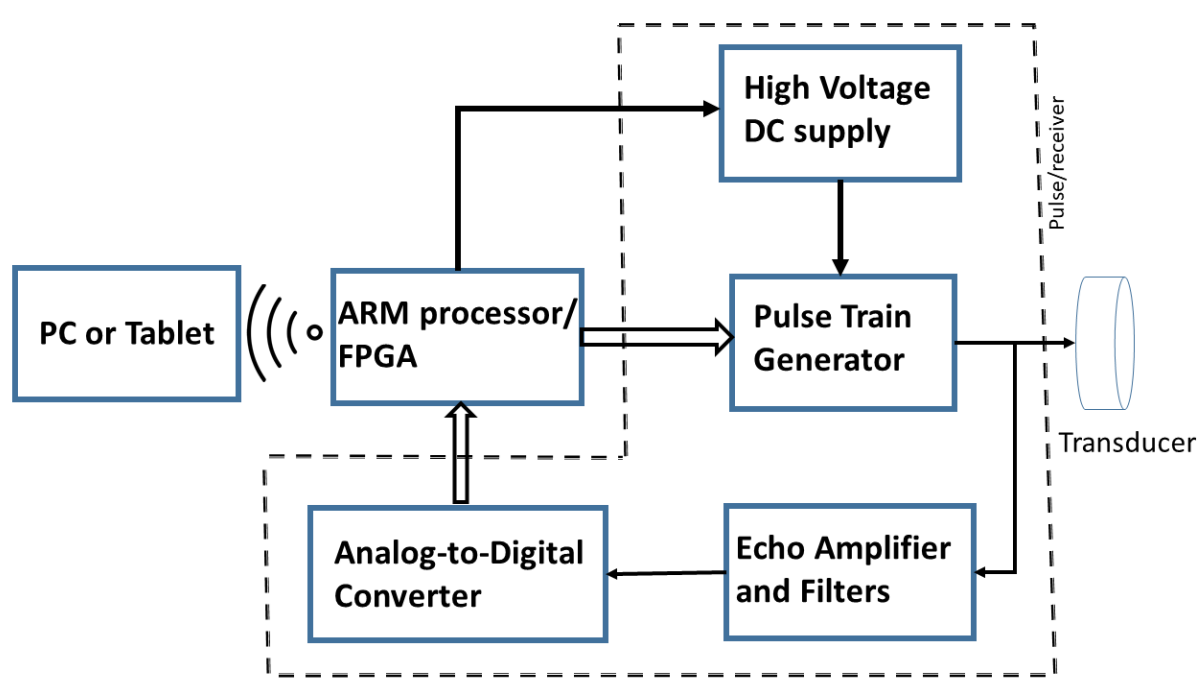

Figure 1. Block diagram of portable ultrasound device. The proposed system will have five major components: Pulse train generator, high voltage amplifiers, A controlling unit consist of a Field Programmable Gate Array (FPGA) and an ARM microcontroller, ultrasound transducer, and an analog-to-digital converter. 
ex vivo tissue types. The best performing speckle feature parameters will be analyzed to find correlations with RF spectrum parameters.

To assess the sensitivity and the specificity of the US system, histopathology data of human kidneys will be used. Autopsied human kidneys with different level of CKD will be collected form the pathologists. Kidney fibrosis will be determined by calculating the percentage of Masson's Trichrome stained area of interstitial fibrosis per total area of kidney tissue. The stained areas will be analyzed with available image software, and the levels of lesion formation will be expressed as percent lesion area per total area. Kidney samples will be scanned using the immersion US probe. Back scattered US signal will be collected and analyzed to determine the sensitivity of $\mathrm{RF}$ and speckle feature parameters to different levels of kidney fibrosis in the autopsied human kidneys.

\section{Proposed clinical study}

In the clinical study, ultrasound imaging will be performed on human subjects with approval from the institutional ethical committee. The standard diagnostic criteria will be applied to diagnose CKD participants and they will be classified into stages (five) according to eGFR by using CKD-EPI equation ${ }^{26}$. The sample size will be based on the uncertainty of textural parameters determined in the experimental work.

B-mode ultrasound imaging will be performed with standard imaging equipment to depict long-axis and transverse views of the kidneys. Kidney longitudinal length (Bi-parietal) will be measured for both kidneys. Additionally, kidney size, cortical echogenicity, parenchymal thickness and cortico-medullary demarcation will be recorded. All clinical results will be normalized to anthropometric features such as body height etc. ${ }^{27}$. The proposed US probe will be used to acquire RF echo data. The RF echo data will be transmitted to a remote server where the RF spectrum will be analyzed to quantitate RF spectrum parameters found in the laboratory experiment.

Anonymized B-mode data will be used to stratify CKD patients using speckle parameters developed in experimental work. The Spearman's correlation test will be used to assess any correlations between the clinical and biochemical data with B-mode speckle parameters. The positive predictive values of the B-mode speckle scoring system and RF spectrum parameters will be compared with the results of the CKD stages. It is expected that the combination of ex vivo and clinical study results will enable us to identify an optimal set of RF spectrum parameters that will be used to diagnose early signs of CKD.

The successful completion of this project will result in, a novel ultrasound parameter of CKD that can detect the disease at early stages and technology to construct a device that can make noninvasive diagnostic measurements of the kidney. A simple diagnostic tool that is portable will have significant impact on the future studies of CKD. This ultrasound device technology that will be developed in this study is potentially patentable and has a commercial value.

\section{Grant information}

Funding by National Research Council, Vidya Mawatha, Sri Lanka (NRC 16-044).

The funders had no role in study design, data collection and analysis, decision to publish, or preparation of the manuscript.
1. Lozano R, Naghavi M, Foreman $\mathrm{K}$, et al:: Global and regional mortality from 235 causes of death for 20 age groups in 1990 and 2010: a systematic analysis for the Global Burden of Disease Study 2010. Lancet. 2012; 380(9859): 2095-128. PubMed Abstract | Publisher Full Text

2. Coresh J, Byrd-Holt D, Astor BC, et al.: Chronic kidney disease awareness, prevalence, and trends among U.S. adults, 1999 to 2000. J Am Soc Nephrol. 2005; 16(1): 180-8.

PubMed Abstract | Publisher Full Text

3. Levey AS, Atkins R, Coresh J, et al:: Chronic kidney disease as a global public health problem: approaches and initiatives - a position statement from Kidney Disease Improving Global Outcomes. Kidney Int. 2007; 72(3): 247-59. PubMed Abstract | Publisher Full Text

4. Jha V, Garcia-Garcia G, Iseki K, et al.: Chronic kidney disease: global dimension and perspectives. Lancet. 2013; 382(9888): 260-72. PubMed Abstract | Publisher Full Text

5. Whitman IR, Feldman HI, Deo R: CKD and sudden cardiac death: epidemiology, mechanisms, and therapeutic approaches. J Am Soc Nephrol. 2012; 23(12): 1929-39.

PubMed Abstract | Publisher Full Text | Free Full Text

6. Global Burden of Disease 2013 Risk Factors Collaborators, Forouzanfar $\mathrm{MH}$, Alexander L, et al.: Global, regional, and national comparative risk assessment of 79 behavioural, environmental and occupational, and metabolic risks or clusters of risks in 188 countries, 1990-2013: a systematic analysis for the Global Burden of Disease Study 2013. Lancet. 2015; 386(10010): 2287-323. PubMed Abstract | Publisher Full Text | Free Full Text

7. Jayasumana C, Gunatilake S, Siribaddana S: Simultaneous exposure to multiple heavy metals and glyphosate may contribute to Sri Lankan agricultural nephropathy. BMC Nephrol. 2015; 16: 103. PubMed Abstract | Publisher Full Text | Free Full Text

8. Jayatilake N, Mendis S, Maheepala $\mathrm{P}$, et al:: Chronic kidney disease of uncertain aetiology: prevalence and causative factors in a developing country. $B M C$ Nephrol. 2013; 14: 180.

PubMed Abstract | Publisher Full Text | Free Full Text

9. Kavvadas P, Dussaule JC, Chatziantoniou C: Searching novel diagnostic markers and targets for therapy of CKD. Kidney Int Suppl (2011). 2014; 4(1): 53-57.

PubMed Abstract | Publisher Full Text | Free Full Text

10. Wong MG, Pollock CA: Biomarkers in kidney fibrosis: are they useful? Kidney Int Suppl (2011). 2014; 4(1): 79-83.

PubMed Abstract | Publisher Full Text | Free Full Text

11. Ju W, Nair V, Smith S, et al.: Tissue transcriptome-driven identification of epidermal growth factor as a chronic kidney disease biomarker. Sci Trans/ Med. 2015; 7(316): 316ra193

PubMed Abstract | Publisher Full Text | Free Full Text

12. Boor $\mathrm{P}$, Ostendorf $\mathrm{T}$, Floege $\mathrm{J}$ : Renal fibrosis: novel insights into mechanisms and therapeutic targets. Nat Rev Nephrol. 2010; 6(11): 643-56. PubMed Abstract | Publisher Full Text

13. Muñoz-Félix JM, González-Núñez M, Martínez-Salgado C, et al:: TGF-ß/BMP proteins as therapeutic targets in renal fibrosis. Where have we arrived after 25 years of trials and tribulations? Pharmacol Ther. 2015; 156: 44-58. PubMed Abstract | Publisher Full Text

14. Diwan A, Wansapura J, Syed FM, et al.: Nix-mediated apoptosis links myocardial 
fibrosis, cardiac remodeling, and hypertrophy decompensation. Circulation. 2008; 117(3): 396-404.

PubMed Abstract | Publisher Full Text | Free Full Text

15. Malatesta-Muncher R, Wansapura J, Taylor M, et al.: Early cardiac dysfunction in pediatric patients on maintenance dialysis and post kidney transplant. Pediatr Nephrol. 2012; 27(7): 1157-64.

PubMed Abstract | Publisher Full Text | Free Full Text

16. Serón D: Risk factors associated with the deterioration of renal function: the role of protocol biopsies. Prilozi. 2007; 28(1): 291-302. PubMed Abstract

17. Wansapura J, Hor KN, Mazur W, et al.: Left ventricular T2 distribution in Duchenne muscular dystrophy. J Cardiovasc Magn Reson. 2010; 12 : 14. PubMed Abstract | Publisher Full Text | Free Full Text

18. Zaffanello M, Piacentini G, Bruno C, et al.: Renal elasticity quantification by acoustic radiation force impulse applied to the evaluation of kidney diseases: a review. J Investig Med. 2015; 63(4): 605-12.

PubMed Abstract | Publisher Full Text

19. Wang L, Xia P, Lv K, et al:: Assessment of renal tissue elasticity by acoustic radiation force impulse quantification with histopathological correlation: preliminary experience in chronic kidney disease. Eur Radiol. 2014; 24(7): 1694-9. PubMed Abstract | Publisher Full Text

20. Takata T, Koda M, Sugihara T, et al.: Renal shear wave velocity by acoustic radiation force impulse did not reflect advanced renal impairment. Nephrology (Carlton). 2016; 21(12): 1056-62.

PubMed Abstract | Publisher Full Text
21. Lizzi FL, Feleppa EJ, Kaisar Alam S, et al.: Ultrasonic spectrum analysis for tissue evaluation. Pattern Recognit Lett. 2003; 24(4-5): 637-58. Publisher Full Text

22. Gao S, Peng Y, Guo H, et al:: Texture analysis and classification of ultrasound liver images. Biomed Mater Eng. 2014; 24(1): 1209-16.

PubMed Abstract | Publisher Full Tex

23. Cao G, Shi $P$, Hu B: Liver fibrosis identification based on ultrasound images. Conf Proc IEEE Eng Med Biol Soc. 2005; 6: 6317-20. PubMed Abstract | Publisher Full Text

24. Layer G, Zuna I, Lorenz A, et al.: Computerized ultrasound B-scan texture analysis of experimental diffuse parenchymal liver disease: correlation with histopathology and tissue composition. J Clin Ultrasound. 1991; 19(4): 193-201.

PubMed Abstract | Publisher Full Text

25. Damerjian V, Tankyevych O, Souag N, et al.: A Speckle characterization methods in ultrasound images - A review. IRBM. 2014; 35(4): 202-13.

Publisher Full Text

26. Levey AS, Stevens $\mathrm{LA}$, Schmid $\mathrm{CH}$, et al:: A new equation to estimate glomerular filtration rate. Ann Intern Med. 2009; 150(9): 604-12.

PubMed Abstract | Publisher Full Text | Free Full Text

27. Lucisano G, Comi N, Pelagi E, et al:: Can renal sonography be a reliable diagnostic tool in the assessment of chronic kidney disease? J Ultrasound Med. 2015; 34(2): 299-306. PubMed Abstract | Publisher Full Text 


\section{Open Peer Review}

\section{Current Peer Review Status:}

\section{Version 2}

Reviewer Report 12 March 2019

https://doi.org/10.5256/f1000research.20082.r44855

(C) 2019 Lucisano G. This is an open access peer review report distributed under the terms of the Creative Commons Attribution License, which permits unrestricted use, distribution, and reproduction in any medium, provided the original work is properly cited.

\section{Gaetano Lucisano}

Imperial College Renal and Transplant Centre, London, UK

No further comments.

Competing Interests: No competing interests were disclosed.

Reviewer Expertise: Ultrasound in Nephrology; Transplant Medicine; Transplant Immunology I confirm that I have read this submission and believe that I have an appropriate level of expertise to confirm that it is of an acceptable scientific standard.

\section{Version 1}

Reviewer Report 23 January 2019

\section{https://doi.org/10.5256/f1000research.15472.r41537}

(c) 2019 Nalesso F. This is an open access peer review report distributed under the terms of the Creative Commons Attribution License, which permits unrestricted use, distribution, and reproduction in any medium, provided the original work is properly cited.

\section{Federico Nalesso}

DIMED - UOC NEFROLOGIA 2- Azienda Ospedaliera, Università di Padova, Padova, Italy

The article introduces a new very interesting ultrasound examination to be used for the study of CKD.

The parameters used to perform the ultrasound measurements to be correlated with renal 
fibrosis appear to be very difficult to measure and operator-dependent. The standardization appears difficult and very difficult to obtain. The vascular component during fibrosis is not taken into consideration and the correlation of the new ultrasound measurement with renal resistance indices is not analyzed.

CKD is reduced to the fibrotic component only, without considering the vascular alterations that underlie interstitial changes.

Is the topic of the opinion article discussed accurately in the context of the current literature?

Partly

Are all factual statements correct and adequately supported by citations?

Partly

Are arguments sufficiently supported by evidence from the published literature?

No

Are the conclusions drawn balanced and justified on the basis of the presented arguments? Yes

Competing Interests: No competing interests were disclosed.

Reviewer Expertise: CKD, AKI, US in AKI/CKD. Critical care nephrology.

I confirm that I have read this submission and believe that I have an appropriate level of expertise to confirm that it is of an acceptable scientific standard.

Reviewer Report 10 January 2019

https://doi.org/10.5256/f1000research.15472.r41738

(C) 2019 Lucisano G. This is an open access peer review report distributed under the terms of the Creative Commons Attribution License, which permits unrestricted use, distribution, and reproduction in any medium, provided the original work is properly cited.

\section{Gaetano Lucisano}

Imperial College Renal and Transplant Centre, London, UK

This is the proposal of a prospective study aimed to assess the ability of a novel portable ultrasound (US) system to estimate the amount of renal fibrosis. The study would first consist in an ex-vivo experiment in which the US system is tested on bovine solid organs (kidney and liver), ultimately leading to the clinical study on human subjects. In the first instance, the novel US system would be employed as a screening tool for the early diagnosis and staging of chronic kidney disease in the rural population of Sri Lanka. 
The idea and purposes are interesting. Few comments:

1. It is not clear whether the data obtained ex-vivo would be compared against histopathology data, as this would be imperative before proceeding with the clinical study. In this case the investigators should make sure that bovine kidneys with different degrees of fibrosis are analysed in order to properly assess the sensitivity and specificity of the US system.

2. It would be interesting to have an estimate of the saved costs of this technique employed for the rural population of Sri Lanka, as the application could be extended to the many other populations across the world with limited access to the healthcare system.

3. In my view, it is important to point out that, if successful, this US system is aimed to flag patients with suspected CKD - who will eventually need a biochemical estimation of the degree of CKD, as I doubt this system would have sufficient sensitivity to warrant a fairly accurate monitoring of the progression of the CKD (changes in the degree of fibrosis detected by the US system would probably correspond to dramatic decreases of the eGFR).

4. In the clinical study, the obtained B-mode parameters should be corrected for anthropometric features, such as the body height. This would strengthen the degree of correlation between CKD stage and US parameters (Lucisano et al., 2015 ${ }^{1}$ ).

\section{References}

1. Lucisano G, Comi N, Pelagi E, Cianfrone $P$, et al.: Can renal sonography be a reliable diagnostic tool in the assessment of chronic kidney disease?. J Ultrasound Med. 2015; 34 (2): 299-306 PubMed Abstract | Publisher Full Text

Is the topic of the opinion article discussed accurately in the context of the current literature?

Yes

Are all factual statements correct and adequately supported by citations? Yes

Are arguments sufficiently supported by evidence from the published literature? Yes

Are the conclusions drawn balanced and justified on the basis of the presented arguments? Partly

Competing Interests: No competing interests were disclosed.

Reviewer Expertise: Ultrasound in Nephrology; Transplant Medicine; Transplant Immunology

I confirm that I have read this submission and believe that I have an appropriate level of expertise to confirm that it is of an acceptable scientific standard, however I have significant reservations, as outlined above. 
Author Response 19 Feb 2019

Janaka Wansapura, University of Colombo, Colombo, Sri Lanka

Response to reviewer:

1) It is not clear whether the data obtained ex-vivo would be compared against histopathology data, as this would be imperative before proceeding with the clinical study. In this case the investigators should make sure that bovine kidneys with different degrees of fibrosis are analysed in order to properly assess the sensitivity and specificity of the US system.

We agree with the reviewer's concerns and have now included a section for sensitivity analysis as follows:

To assess the sensitivity and the specificity of the US system, histopathology data of human kidneys will be used. Autopsied human kidneys with different level of CKD will be collected form the pathologists. Kidney fibrosis will be determined by calculating the percentage of Masson's Trichrome stained area of interstitial fibrosis per total area of kidney tissue. The stained areas will be analyzed with available image software, and the levels of lesion formation will be expressed as percent lesion area per total area. Kidney samples will be scanned using the immersion US probe. Back scattered US signal will be collected and analyzed to determine the sensitivity of RF and speckle feature parameters to different levels of kidney fibrosis in the autopsied human kidneys.

2) It would be interesting to have an estimate of the saved costs of this technique employed for the rural population of Sri Lanka, as the application could be extended to the many other populations across the world with limited access to the healthcare system.

The following text was added:

It is estimated that a prototype of the proposed US system can be built for approximately USD 1400.

3) In my view, it is important to point out that, if successful, this US system is aimed to flag patients with suspected CKD - who will eventually need a biochemical estimation of the degree of CKD, as I doubt this system would have sufficient sensitivity to warrant a fairly accurate monitoring of the progression of the CKD (changes in the degree of fibrosis detected by the US system would probably correspond to dramatic decreases of the eGFR)

We understand the reviewer's concern regarding the sensitivity of the proposed US system. The proposed clinical study, by way of testing the hypothesis of this paper, is only aimed at establishing a novel set of US based markers for kidney fibrosis. To study the sensitivity and specificity of these makers in relation to CKD, a rigorous clinical study must be performed and the data must be corroborated with established markers such as eGFR. We think such a study is beyond the scope of this hypothesis paper. 
4) In the clinical study, the obtained B-mode parameters should be corrected for anthropometric features, such as the body height. This would strengthen the degree of correlation between CKD stage and US parameters (Lucisano et al., 2015 ${ }^{1}$ ).

As proposed in this study, both ex vivo and clinical study results will be used to identify optimal set of RF spectrum parameters that will lead to identifying CKD at early stages. As Lucisano et al. observed, all clinical results such as one-axis and transverse views of the kidneys, Kidney longitudinal length (Bi-parietal), kidney size, cortical echogenicity, parenchymal thickness and cortico-medullary demarcation, etc. will be corrected for anthropometric features such as body height etc.

The following text was added to the manuscript: All clinical results will be normalized for anthropometric features such as body height etc. (27).

Competing Interests: No competing interests were disclosed.

The benefits of publishing with F1000Research:

- Your article is published within days, with no editorial bias

- You can publish traditional articles, null/negative results, case reports, data notes and more

- The peer review process is transparent and collaborative

- Your article is indexed in PubMed after passing peer review

- Dedicated customer support at every stage

For pre-submission enquiries, contact research@f1000.com 